International Mathematical Forum, Vol. 9, 2014, no. 7, 325 - 333

HIKARI Ltd, www.m-hikari.com

http://dx.doi.org/10.12988/imf.2014.411

\title{
A Reduced Computational Complexity Strategy for the Magnus-Derek Game
}

\author{
Zhivko Nedev \\ Zaman University, Phnom Penh - Cambodia \\ Copyright (C) 2014 Zhivko Nedev. This is an open access article distributed under the Creative \\ Commons Attribution License, which permits unrestricted use, distribution, and reproduction in \\ any medium, provided the original work is properly cited.
}

\begin{abstract}
We analyze the Magnus-Derek game, a two-player game played on a round table having $n$ positions. The players jointly control the movement of a token. One player aims to minimize the number of positions visited, and the other aims to maximize this quantity. We give an $f^{*}(n)+\left\lceil\log _{2}(m-1)\right\rceil f^{*}(m)$ round strategy, where $p$ is the smallest odd prime factor of $n, m=\frac{n}{p}$, and $f^{*}$ is a function yielding the number of positions visited when both players play optimally. This strategy requires significantly less computation time than the previously published strategies.
\end{abstract}

\section{Introduction}

In this paper, we further analyze a two-player game that we call the Magnus-Derek game [4]. The game is played on a circular table with $n$ positions labeled consecutively from 0 to $n-1$ in clockwise direction. A single token is initially placed at position 0. A round consists of the first player, Magnus (from magnitude) choosing an integer magnitude $0<\ell \leq \frac{n}{2}$, followed by the second player, Derek (from $d i$ rection) choosing whether the token will move clockwise or counter-clockwise. The token is then moved $\ell$ positions in the chosen direction. Magnus' goals are first to maximize the total number of positions visited by the token over the course of the game and second, to minimize the number of rounds. Derek aims to minimize the number of positions visited.

We previously obtained an expression for the total number of positions visited when both players play optimally $[3,4]$, and the following theorem was given. 
Theorem 1.1: (from [4]) Let $f^{*}(n)$ denote the total number of positions visited when both players play optimally. If $n=2^{k}$ for some integer $k$, then $f^{*}(n)=n$. Otherwise, let $p$ be the smallest odd prime factor of $n$. Then $f^{*}(n)=\frac{(p-1) n}{p}$.

The sequence $\left\{f^{*}(n)\right\}_{n=1}^{\infty}=(1,2,2,4,4,4,6,8,6,8, \ldots)$ diverges non-monotonically and non-linearly to $\infty[4]$. This sequence was put in the Encyclopedia of Integer Sequences [6] after [3, 4] were published.

Several papers have analyzed the number of rounds required for Magnus to visit $f^{*}(n)$ positions. The original strategy by Nedev and Muthukrishnan required $O\left(n^{2}\right)$ rounds [4]. A recent paper by Hurkens, Pendavingh, and Woeginger requires $O(n \log (n))$ rounds [2]. In a further paper, Nedev gives a strategy in which only $3(n-1)$ rounds - the optimal number, up to a constant factor - are needed [5]. A similar result is also present in a paper by Chen, Lin, Shieh, and Tsai [1].

In these previous analyses, no attention was given to the computational complexity of the strategies. Here we will give a strategies that is more computationally efficient at each round than those in the above papers. The number of rounds required will be $f^{*}(n)+\left\lceil\log _{2}(m-1)\right\rceil f^{*}(m)$, where $p$ is the smallest odd prime factor of $n$ and $m=\frac{n}{p}$, similar to Hurkens, Pendavingh, and Woeginger's result.

In this paper as in all previous papers, we continue to ignore the time needed to compute the smallest prime $p$ dividing $n$. It is still unknown if $n$ can be factorized in $O(\operatorname{polylog}(n))$ and for big $n$, currently the computation time of our strategy as well as any of the previously existing would be dominated by the time to factor $n$.

Although the game was invented to model a practical situation in computer networks, now the game has "its own life." Here we give shortened the original reason for inventing the game.

\section{Motivation}

A mobile agent (MA) is an autonomous computer program possessing the ability to move between hosts in a network. The Vector game models the case of an MA exploring a network with a ring topology where the labels "clockwise" and "counter-clockwise" are not consistent among hosts. That is, there are $n$ hosts $H_{0}, H_{1}, \ldots, H_{n-1}$, and for each $i, H_{i}$ is connected to $H_{i+1}(\bmod n)$ and $H_{i-1}(\bmod n)$. But when the MA visits a host, it cannot distinguish between the host's two neighbours.

Before moving, the MA can predetermine the magnitude of its travel (i.e. how many hosts it will pass through), but it cannot control the initial direction of its motion around the ring. Once it begins to move, it will keep traveling in the same direction until it stops. The MA is only able to explore hosts at which it stops.

We give an algorithmic strategy ensuring that the MA explores as many hosts as possible under the worst case, i.e. that the direction of motion is chosen maliciously to minimize the number of hosts explored, taking into account the secondary goals 
of minimizing the number of moves and the computation time required by the MA. We formulate the problem as an adversarial game between Magnus, representing the MA, and Derek, representing the malicious adversary.

In this game, optimal strategies for both players depend on the factors of $n$, the table size. We hope that as we further develop our understanding of strategies for the game it might yield insight into the problem of integer factorization.

\section{A reduced-computation strategy}

We will present a strategy for Magnus to visit $f^{*}(n)$ positions in $f^{*}(n)+\left\lceil\log _{2}(m-1)\right\rceil f^{*}(m)$ rounds, where $p$ is the smallest odd prime factor of $n$ and $m=\frac{n}{p}$. This strategy requires less computation time at each round than the existing strategies.

We will use $\mathbb{Z}_{n}$ (the ring of integers modulo $n$ ) to represent a table of size $n$. Let $K \in \mathbb{Z}_{n}$ denote the position of the token at the beginning of a round. A round then consists of Magnus choosing a magnitude $0<\ell \leq \frac{n}{2}$, Derek choosing a direction + or - (where the positive direction corresponds to "clockwise"), and the token moving to $K \pm \ell(\bmod n)$.

When we refer to the distance between two positions $A, B \in \mathbb{Z}_{n}$, we will mean the following: Let $0<\delta<n$ be such that $A+\delta \equiv B(\bmod n)$ or $A-\delta \equiv B$ $(\bmod n)$. Then the distance is $\min \{\delta, n-\delta\}$.

We begin by considering the case when $n$ is odd. The core of our strategy for odd $n$ is a technique for Magnus to visit one of a pair of target positions.

\subsection{Reaching one of two target positions in at most $\left\lceil\log _{2}(n-1)\right\rceil$ rounds}

Let the table size $n>2$ be odd, and let $A, B \in \mathbb{Z}_{n}$ with $A<B$ and $\operatorname{gcd}(B-A, n)=1$. Let $\delta:=B-A$, let $K \notin\{A, B\}$ be the current position of the token, and set $h:=\left\lceil\log _{2}(n-1)\right\rceil$. We give a technique for Magnus to move the token to one of $\{A, B\}$ in at most $\left\lceil\log _{2}(n-1)\right\rceil$ rounds.

One idea is to be used by all strategies for Magnus: if the token is equidistant from two positions (with distance $d$ from each) then, regardless of the direction chosen by Derek, Magnus can cause the token to move to one of those positions by choosing magnitude $d$. We will also use this idea by defining a nested sequence of subsets $\{A, B\}=F_{0} \subseteq F_{1} \subseteq \cdots \subseteq F_{h}=\mathbb{Z}_{n}$ with the property that for each $0<k \leq h$, every position in $F_{k} \backslash F_{k-1}$ is equidistant from a pair of positions in $F_{k-1}$. Thus if $K \in F_{k} \backslash F_{k-1}$ for some $k$, then Magnus can move the token to a position in $F_{k-1}$ in one round. It follows that since $K \in \mathbb{Z}_{n}=F_{h}$, Magnus can move the token to one of $\{A, B\}$ in at most $\left\lceil\log _{2}(n-1)\right\rceil$ rounds.

Since $n$ is odd, $2^{-1} \in \mathbb{Z}_{n}^{*}$. Set $\delta_{k}:=2^{-k} \delta$ for $0 \leq k \leq h$; then $\operatorname{gcd}\left(\delta_{k}, n\right)=1$, and $\delta_{k}$ is a generator for $\mathbb{Z}_{n}$. 
Definition 3.1: For $0 \leq k \leq h$, we define $F_{k}$ to be the sequence $\left\{A+i \delta_{k}\right\}_{i=0}^{2^{k}} \subseteq \mathbb{Z}_{n}$. Note that we will sometimes use $F_{k}$ to mean the set of elements of the sequence, rather than the sequence itself.

Intuitively, $F_{k}$ consists of $F_{k-1}$ as well the midpoints between consecutive elements of $F_{k-1}$. For example, let $c:=2^{-1}(A+B)$ be equidistant from $A$ and $B$. Then $F_{0}=\{A, B\}, F_{1}=\{A, c, B\}$ and $F_{2}=\left\{A, 2^{-1}(A+c), c, 2^{-1}(c+B), B\right\}$.

Remark 3.2: In the 3(n-1)-round strategy [5], the map

$M(S):=\left\{z \in \mathbb{Z}_{n}:\right.$ there exist $x, y \in S$ such that $\left.2 z \equiv x+y \quad(\bmod n)\right\} \quad$ for $S \subseteq \mathbb{Z}_{n}$ was defined, and called the set of all middles between pairs of elements of $S$. The notation $M^{(i)}(S)$ was used to denote the recursive application to $S$ of the map $M, i$ times.

For odd $n$, definition 3.1 corresponds to this map. That is, for each $0 \leq k \leq h$, the set of elements of $F_{k}$ is equal to $M^{(k)}(\{A, B\})$.

Lemma 3.3: $\{A, B\}=F_{0} \subseteq F_{1} \subseteq \cdots \subseteq F_{h}=\mathbb{Z}_{n}$, and for $k<h$, the elements of $F_{k}$ are distinct.

Proof. Since the sequence $F_{h}$ contains $2^{h}+1$ elements, we have that $F_{h}=\mathbb{Z}_{n}$ (considering $F_{h}$ here as the set of elements). The other containments follow immediately from the definition of $F_{k}$. For $k<h$ we have that $2^{k}+1<n$, and since $\delta_{k}$ is a generator all elements of $F_{k}$ are distinct.

Definition 3.4: Let $e \in \mathbb{Z}_{n}$. We define $\alpha(e)$ to be the smallest integer $k$ such that $e \in F_{k}$. Since $F_{h}=\mathbb{Z}_{n}, \alpha(e)$ is well-defined and $\alpha(e) \leq h$.

Lemma 3.5: Let $e \in \mathbb{Z}_{n}$ and $1 \leq k<h$. Then $\alpha(e)=k$ if and only if $e$ is an odd-indexed element of the sequence $F_{k}$. Furthermore, if $\alpha(e)=h$ then $e$ is an odd-indexed element of $F_{h}$.

Proof. Let $1 \leq k<h$, and let $e$ be an odd-indexed element of $F_{k}$. That is to say, $e=A+i \delta_{k}$ for some $0 \leq i \leq 2^{k}, i$ odd. Suppose that $\alpha(e)<k$. Then $e \in F_{\ell}$ for some $\ell<k$ and we may write $e=A+i^{\prime} \delta_{\ell} \equiv A+\left(2^{k-\ell} i^{\prime}\right) \delta_{k}(\bmod n)$ for some $0 \leq i^{\prime} \leq 2^{\ell}$. But $\operatorname{gcd}\left(\delta_{k}, n\right)=1$, so there exists a unique solution for $x$ in $e \equiv A+x \delta_{k}$ $(\bmod n)$. Thus we have $i=2^{k-\ell} i^{\prime}$, contradicting $i$ being odd. So $e \notin F_{\ell}$ for any $\ell<k$, and thus $\alpha(e)=k$.

Conversely, let $1 \leq k \leq h$, and let $\alpha(e)=k$. By definition, $e \in F_{k} \backslash F_{k-1}$. Suppose $e$ has an even index in $F_{k}$. Then $e=A+\left(2^{\ell} i\right) \delta_{k}$ for some odd $i$ and integer $\ell \geq 1$ with $0 \leq 2^{\ell} i \leq 2^{k}$. But then $e=A+i \delta_{k-\ell}$ with $0 \leq i \leq 2^{k-\ell}$, and so $e \in F_{k-\ell}$ contradicting $i \notin F_{k-1}$. Thus $e$ is an odd-indexed element of $F_{k}$.

Lemma 3.6: Let $e \in \mathbb{Z}_{n}$. If $\alpha(e)>0$, then e is equidistant (with distance $\min \left\{\delta_{\alpha(e)}, n-\right.$ $\left.\left.\delta_{\alpha(e)}\right\}\right)$ from two distinct positions $e^{\prime}, e^{\prime \prime} \in F_{\alpha(e)-1}$. 
Proof. Let $\alpha(e)>0$, and set $k:=\alpha(e)$. By definition, $e \in F_{k} \backslash F_{k-1}$. By Lemma 3.5, $e=A+(2 i-1) \delta_{k}$ for some $1 \leq i \leq 2^{k-1}$. Let $e^{\prime}=A+(2 i-2) \delta_{k}=A+(i-1) \delta_{k-1}$ and $e^{\prime \prime}=A+(2 i) \delta_{k}=A+i \delta_{k-1}$. Then $e^{\prime}, e^{\prime \prime} \in F_{k-1}$, and by Lemma 3.3 they are distinct. Furthermore, $e$ has distance $\min \left\{\delta_{k}, n-\delta_{k}\right\}$ from each of these points. Thus $e$ is the midpoint between two distinct positions $e^{\prime}$ and $e^{\prime \prime}$ in $F_{\alpha(e)-1}$, and has distance $\min \left\{\delta_{\alpha(e)}, n-\delta_{\alpha(e)}\right\}$ from each of these positions.

Magnus begins by precomputing $\delta_{h-1}$ and $\delta_{h}$ as follows. He computes $2^{-1}$ (as $\left.\left\lceil\frac{n}{2}\right\rceil=\frac{n+1}{2}\right)$ using $O(\log (n))$ bit-operations, $2^{h-1}$ using $O(\log (n))$ bit-operations, and $2^{-(h-1)}$ and $2^{-h}$ using $O\left((\log (n))^{2}\right)$ bit-operations [7]. (Note that these computations need happen only once, at the very beginning of the game.) He then uses the Extended Euclidean Algorithm to solve for $x$ in $K \equiv A+x \delta_{h-1}(\bmod n)$ using $O\left((\log (n))^{2}\right)$ bit-operations. (A unique solution exists since $\operatorname{gcd}\left(\delta_{h-1}, n\right)=1$.) Since $K \notin\{A, B\}$, we have that $x \neq 0, x \neq 2^{h-1}$.

If $2^{h-1}<x<n$, an initial step is required. In this case $K \notin F_{h-1}$, so $\alpha(K)=h$. We have that $K \equiv A+x \delta_{h-1} \equiv A+2 x \delta_{h}$. Then by Lemma 3.6, there exist positions $K^{\prime}, K^{\prime \prime} \in F_{h-1}$, where $K^{\prime}=A+(2 x-1) \delta_{h}=A+\left(x-2^{-1}\right) \delta_{h-1}$ and $K^{\prime \prime}=A+(2 x+1) \delta_{h}=A+\left(x+2^{-1}\right) \delta_{h-1}$. Magnus chooses the magnitude $\delta_{h}$, and the token moves to one of these positions. We then set $K$ to the new position of the token, and set $x$ to $\left(x+2^{-1}(\bmod p)\right)$ or $\left(x-2^{-1}(\bmod p)\right)$ as appropriate. Now $\alpha(K)<h$ and $x<2^{h-1}$.

At this point $0<x<2^{h-1}$. We set $\ell:=h-1$, and begin the repetitive part of the algorithm.

We have that $K \equiv A+x \delta_{\ell}(\bmod n)$. (Note that we already have $\delta_{\ell}$ calculated at this point.) Using $O(\log (n))$ bit-operations, we factor $x=2^{j} y$ such that $y$ is odd [7]. Then $K \equiv A+y \delta_{\ell-j}$ and so $K \in F_{\ell-j}$. It follows from Lemma 3.5 that $\alpha(K)=\ell-j$. By Lemma 3.6, there exist points $K^{\prime}, K^{\prime \prime} \in F_{(\ell-j)-1}$, each with distance $\min \left\{\delta_{\ell-j}, n-\delta_{\ell-j}\right\}$ from $K$. Magnus now calculates $\delta_{\ell-j}=\delta_{\ell} 2^{j} \in \mathbb{Z}_{n}$ using $O(j \log (n))$ bit-operations [7]. He then chooses the magnitude $\delta_{\ell-j}$, and the token moves to a position $K_{\text {new }} \in F_{(\ell-j)-1}$. Note that $\alpha\left(K_{\text {new }}\right)<\alpha(K)$. Futhermore, $K_{\text {new }}$ is either $A+(y+1) \delta_{\ell-j}$ or $A+(y-1) \delta_{\ell-j}$. We therefore set $K$ to $K_{\text {new }}$, set $x$ to $(y+1)$ or $(y-1)$ as appropriate, and set $\ell$ to $\ell-j$. We repeat the steps in this paragraph until $K \in\{A, B\}$.

Since $\alpha(K) \leq h$ initially, this algorithm will terminate in at most $\left\lceil\log _{2}(n-1)\right\rceil$ rounds. Magnus' computations at each round require at most $O(\log (n))$ bit-operations, so overall at most $O\left((\log (n))^{2}\right)$ bit-operations are required, including the precomputations.

We claim that this algorithm for Magnus is optimal in the number of rounds. That is, if $K_{\text {init }}$ is the position of the token at some point during the game, then Derek can prevent Magnus from reaching $F_{0}$ in fewer than $\alpha\left(K_{\text {init }}\right)$ rounds. Suppose otherwise. Then there is a round where $\alpha(K)=k$ for some $2 \leq k \leq h$ but $K_{\text {new }} \in$ $F_{k-2}$, i.e. Magnus moves the token from $F_{k}$ to $F_{k-2}$ (or even further) in one round. 
Then there must exist two positions $K^{\prime}, K^{\prime \prime} \in F_{k-2}$, each equidistant from $K$, such that $K_{\text {new }}$ is one of these positions. Writing $K^{\prime} \equiv A+i^{\prime} \delta_{k-2}(\bmod n)$ for some $0 \leq i^{\prime} \leq 2^{k-2}$ and $K^{\prime \prime} \equiv A+i^{\prime \prime} \delta_{k-2}(\bmod n)$ for some $0 \leq i^{\prime \prime} \leq 2^{k-2}$ we have that $K \equiv 2^{-1}\left(K^{\prime}+K^{\prime \prime}\right)=A+\left(i^{\prime}+i^{\prime \prime}\right) \delta_{k-1}(\bmod n)$ with $0 \leq i^{\prime}+i^{\prime \prime} \leq 2^{k-1}$, so $K \in F_{k-1}$ contradicting $\alpha(K)=k$. Thus $\alpha\left(K_{\text {init }}\right)$ rounds are required.

\subsection{Magnus' strategy on an odd-sized table}

We are now able to give Magnus' strategy for playing on a table with an odd number of positions.

Let the table size $n$ be odd, and let $p$ be the smallest prime factor of $n$. We partition the $n$ positions on the table into the subsets

$$
S_{i}=\left\{e \in \mathbb{Z}_{n}: i p \leq e<(i+1) p\right\} \text { for } i \in\left\{0,1, \ldots, \frac{n}{p}-1\right\} .
$$

While there exists a subset $S_{i}$ containing a pair of unvisited positions $A, B \in S_{i}$, Magnus employs the above algorithmic technique (section 3.1) to visit one of the positions in at most $\left\lceil\log _{2}(n-1)\right\rceil$ rounds with $O\left((\log (n))^{2}\right)$ computation time. (Note that by the definition of $S_{i}$ we have that $0 \leq|B-A| \leq p-1$. Since $p$ is the smallest prime factor of $n$ it follows that $\operatorname{gcd}(B-A, n)=1$, satisfying the condition at the beginning of section 3.1.) When there is no remaining subset containing two unvisited positions, Magnus is done.

Since at most one position per subset is left unvisited, Magnus has visited $n-\frac{n}{p}=$ $f^{*}(n)$ positions. Since Magnus employs the algorithm in section 3.1 once for each position visited, he uses at most $f^{*}(n)\left\lceil\log _{2}(n-1)\right\rceil$ rounds and $O\left(f^{*}(n)\left(\log _{2}(n)\right)^{2}\right)$ computation time (plus the computation time to find a subset $S_{i}$ containing a pair of unvisited positions). We therefore obtain the following result.

Lemma 3.7: When the table size $n$ is odd, there exists a strategy for Magnus to visit $f^{*}(n)$ positions in at most $f^{*}(n)\left\lceil\log _{2}(n-1)\right\rceil$ rounds.

Remark 3.8: We will later consider the case where Magnus chooses a particular pair of unvisited positions and, at the cost of additional computation time, achieves a reduced number of rounds.

This technique for playing on odd-sized tables offers an improvement in computational complexity over the corresponding technique in the $3(n-1)$-round strategy (When $n$ is prime [5]). In that strategy, a large set $\mathbb{A}$ of unvisited positions was used instead of an unvisited pair $\{A, B\}$. At each round, Magnus had to calculate the smallest integer $k$ such that $M^{(k)}(\mathbb{A})=\mathbb{Z}_{n}$, and for each position $z \in M^{(i)}(\mathbb{A}) \backslash M^{(i-1)}(\mathbb{A})(1 \leq i \leq k)$ he needed to record a pair of positions $x, y \in M^{(i-1)}(\mathbb{A})$ such that $z$ is equidistant from $x$ and $y$. This required that he exhaustively compute $M(\mathbb{A}), M^{(2)}(\mathbb{A}), M^{(3)}(\mathbb{A}), \ldots$ until he reached $\mathbb{Z}_{n}$, and that he 
had sufficient storage space to record the equidistant pairs of positions. In contrast, the strategy given here requires only the Extended Euclidean Algorithm, factoring out powers of two, and basic algebraic calculations.

\subsection{Overall strategy for an arbitrary table size}

We now explain Magnus' strategy for playing on a table of arbitrary size. We begin by quoting previous results for playing on a table whose size is a power of two or whose size is composite.

Theorem 3.9: (from [4]) If the table size $n=2^{k}$ for some integer $k$, then $f^{*}(n)=$ $n$, and there exists a strategy for Magnus to visit all $n$ positions in $n-1$ rounds.

Lemma 3.10 (Decomposition): (from [5]) Let the table size be $n=a b$, where $a$ and $b$ are integers greater than 1. Suppose that on a table of size a, Magnus has a strategy $\alpha$ to visit $f^{*}(a)$ positions in $r(a)$ rounds, and that on a table of size $b$, Magnus has a strategy $\beta$ to visit $f^{*}(b)$ positions in $r(b)$ rounds. Then on the table of size $n$, Magnus is able to visit $f^{*}(a) f^{*}(b)$ positions in $r(b)+r(a) f^{*}(b)$ rounds.

The proof of the above lemma is given in appendix A.

We can now give a result for an arbitrary table of size $n$ that uses the strategy from section 3.2 .

Theorem 3.11: Let the table size be $n=2^{k} m$ where $m$ is odd. Let $p$ be the smallest prime factor of $m$. The Magnus can visit $f^{*}(n)$ positions in at most $f^{*}(n)+\left\lceil\log _{2}(m-1)\right\rceil f^{*}(m)$ rounds.

Proof. By Theorem 3.9 there exists a strategy $\alpha$ such that Magnus can visit $2^{k}$ positions on a table of size $2^{k}$ in $2^{k}-1$ rounds.

By Theorem 1.1 we have that $f^{*}(m)=\frac{(p-1) m}{p}$. It follows from Lemma 3.7 that there exists a strategy $\beta$ such that Magnus can visit $\frac{(p-1) m}{p}$ positions on a table of size $m$ in at most $\frac{(p-1) m}{p}\left\lceil\log _{2}(m-1)\right\rceil$ rounds.

We now apply Lemma 3.10, letting $a=2^{k}$ and $b=m$, and find that Magnus can visit $f^{*}\left(2^{k}\right) f^{*}(m)=f^{*}(n)$ positions in at most $\frac{(p-1) m}{p}\left\lceil\log _{2}(m-1)\right\rceil+\left(2^{k}-1\right) \frac{(p-1) m}{p}$ rounds. But

$$
\begin{aligned}
& \frac{(p-1) m}{p}\left\lceil\log _{2}(m-1)\right\rceil+\left(2^{k}-1\right) \frac{(p-1) m}{p} \\
& \leq \frac{(p-1) m}{p}\left\lceil\log _{2}(m-1)\right\rceil+\frac{(p-1)\left(2^{k} m\right)}{p} \\
& =\left\lceil\log _{2}(m-1)\right\rceil f^{*}(m)+f^{*}(n) .
\end{aligned}
$$




\section{Open problems}

- Can we find a nontrivial lower bound for the number of rounds needed by Magnus to visit $f^{*}(n)$ positions?

- Is there a strategy for Derek that maximizes the number of rounds Magnus requires?

- If Magnus has a target set of positions that he wishes to visit, how do we adapt his strategy to visit as many as possible of them in as few rounds as possible?

\section{Acknowledgments}

I am grateful Noam Sturmwind for editing assistance.

\section{A Proof of the Decomposition Lemma}

Lemma (Decomposition): (from [5]) Let the table size be $n=a b$, where $a$ and $b$ are integers greater than 1. Suppose that on a table of size a, Magnus has a strategy $\alpha$ to visit $f^{*}(a)$ positions in $r(a)$ rounds, and that on a table of size $b$, Magnus has a strategy $\beta$ to visit $f^{*}(b)$ positions in $r(b)$ rounds. Then on the table of size $n$, Magnus is able to visit $f^{*}(a) f^{*}(b)$ positions in $r(b)+r(a) f^{*}(b)$ rounds.

Proof. We partition the table positions (i.e. the elements of $\mathbb{Z}_{n}$ ) into the $b$ congruence classes modulo $b$, each of size $a$ :

$$
[i]_{b}=\{i+j b: 0 \leq j \leq a-1\} \quad \text { for } 0 \leq i \leq b-1 .
$$

We first note that Magnus can restrict his play to positions in a particular class. Let $K$ denote the current position of the token, and suppose that $K \in[i]_{b}$ for some $0 \leq i \leq b-1$. Magnus can then play as follows on an imaginary table of size $a$ whose positions are the elements of $[i]_{b}$. On each round, Magnus employs strategy $\alpha$ to calculate a magnitude $\ell$ as if the table size were $a$, but then chooses $b \ell$ as the actual magnitude to use. Then the new position of the token will be $K \pm b \ell \in[i]_{b}$, and so the token will remain within the class.

Magnus' overall strategy will be to repeatedly move the token to an unvisited class, and then, by restricting his play to positions within that class, use strategy $\alpha$ to visit $f^{*}(a)$ positions within that class in $r(a)$ rounds.

Now suppose that $K \in[i]_{b}$ for some $i$, and notice that for any magnitude $\ell$, $K \pm \ell \in[i \pm \ell(\bmod b)]_{b}$. Magnus can use this idea to play on an imaginary table of size $b$ whose positions correspond to the $b$ congruence classes. By employing strategy $\beta$ on this imaginary table, Magnus will visit $f^{*}(b)$ different classes in $r(b)$ rounds. Each time the token lands in an unvisited class, Magnus temporarily restricts his 
play to that class, and employs strategy $\alpha$ until he has visited $f^{*}(a)$ positions within that class. He then continues with strategy $\beta$ until he reaches another unvisited class.

Since Magnus can visit $f^{*}(b)$ classes in $r(b)$ rounds, and can visit $f^{*}(a)$ positions in $r(a)$ rounds within each class, the total number of positions visited will be $f^{*}(a) f^{*}(b)$ and the total number of rounds will be $r(b)+r(a) f^{*}(b)$.

\section{References}

[1] Li-Jui Chen, Jinn-Jy Lin , Min-Zheng Shieh, Shi-Chun Tsai. More on the MagnusDerek game, Theoretical Computer Science, Vol. 412, Issues 45, 4 Feb 2011, Pages 339344, http://dx.doi.org/10.1016/j.tcs.2010.09.031.

[2] Hurkens, Cor A.J., Pendavingh, Rudi A., and Woeginger, Gerhard J. The MagnusDerek game revisited, Information Processing Letters, Volume 109, Issue 1(2008) 38-40, http://dx.doi.org/10.1016/j.ipl.2008.08.007.

[3] Nedev, Zhivko, Universal Sets and the Vector Game, INTEGERS: The Electronic Journal of Combinatorial Number Theory, 2008, (8), \#A45.

[4] Nedev, Zhivko and Muthukrishnan, S., The Magnus-Derek Game, Theoretical Computer Science, 2008, Volume 393, Issues 1-3, Pages 124-132, DOI: http://dx.doi.org/10.1016/j.tcs.2007.11.016.

[5] Nedev, Zhivko, An $O(n)$-Round Strategy for the Magnus-Derek Game, Algorithms 2010, 3(3), Pages 244-254, DOI: http://dx.doi.org/10.3390/a3030244

[6] Sloane, N. On-line Encyclopedia of Integer Sequences.

http://www.research.att.com/ ${ }^{n j a s / s e q u e n c e s / ~}$

[7] Bach, Eric and Shallit, Jeffrey, Algorithmic number theory. Vol. 1. Efficient algorithms, Foundations of Computing Series. MIT Press, Cambridge, MA, 1996.

Received: January 3, 2014 